\title{
Fluorescence of crystalline 4-(dimethylamino)benzonitrile. Absence of dual fluorescence and observation of single-exponential fluorescence decays
}

\author{
Attila Demeter ${ }^{1}$, Klaas A. Zachariasse * \\ Max-Planck-Institut für biophysikalische Chemie, Spektroskopie und Photochemische Kinetik, 37070 Göttingen, Germany
}

Received 4 September 2003; in final form 17 September 2003

Published online: 9 October 2003

\begin{abstract}
The fluorescence spectrum of crystals grown from newly synthesized 4-(dimethylamino)benzonitrile (DMABN), measured from 25 down to $-112{ }^{\circ} \mathrm{C}$, consists of a single emission band originating from a locally excited (LE) state. The fluorescence decay of the DMABN crystals is single exponential at all temperatures investigated. These results show that intramolecular charge transfer (ICT) does not occur in crystalline DMABN. The additional red-shifted emission bands and multiexponential fluorescence decays previously reported for DMABN crystals are attributed to a minor amount of the impurity 4-(dimethylamino)benzaldehyde, the synthetic precursor of commercial DMABN.

(c) 2003 Elsevier B.V. All rights reserved.
\end{abstract}

\section{Introduction}

Recently, we reported fluorescence spectra and decay curves of crystalline 4-(dimethylamino)benzonitrile (DMABN) [1]. At room temperature, the DMABN crystals mainly showed locally excited (LE) fluorescence. In addition, a relatively weak red-shifted structured emission band was observed, in agreement with the spectrum of pow-

\footnotetext{
${ }^{*}$ Corresponding author. Fax: +49-551-201-1501.

E-mail address: kzachar@gwdg.de (K.A. Zachariasse).

${ }^{1}$ On leave from the Institute of Chemistry, Chemical Research Center, Hungarian Academy of Sciences, P.O. Box 17, 1525 Budapest, Hungary.
}

dered DMABN crystals reported in the literature [2]. Upon lowering the temperature, the new redshifted band strongly increased in absolute and relative intensity, reaching at $-110^{\circ} \mathrm{C}$ an intensity ratio of 23 with respect of the fluorescence band attributed to the LE state [1]. Evidence for an intramolecular charge transfer (ICT) fluorescence was not found. It was suggested that the shape of the additional emission band in the spectrum resembles that of DMABN phosphorescence in lowtemperature glassy media, red-shifted by around $2800 \mathrm{~cm}^{-1}$. Such strongly red-shifted structured and quenched luminescence bands were not observed with crystals of the related aminobenzonitriles 4-(methylamino)benzonitrile (MABN) and 
4-(diisopropylamino)benzonitrile (DIABN) at room temperature [1].

The DMABN used in the experiments reported in [1] was purified by sublimation. As impurities can act as very efficient traps in crystal luminescence $[3,4]$, a more extensive purification of DMABN was undertaken, by employing chromatography and zone refining, as described in the experimental part, in order to verify the nature of the red-shifted emission bands. The luminescence spectra of crystals of DMABN purified in this manner, still showed the presence of an extra band next to the LE fluorescence [5], similar to that obtained without the additional purification.

Commercial DMABN contains a relatively large amount (at least $1 \%$, see Section 2) of its synthetic precursor 4-(dimethylamino)benzaldehyde (DMBA). In order to avoid possible contamination with DMBA, which even in minute amounts can act as a trap in crystal luminescence studies as mentioned above, DMABN was synthesized starting from 4-bromo- $N, N$-dimethylaniline. The results of an investigation of the photostationary and time-resolved fluorescence of crystals grown from this newly synthesized DMABN are reported here.

\section{Experimental}

DMABN was synthesized from 4-bromo- $N, N$ dimethylaniline (Aldrich) in a reaction with $\mathrm{CuCN}$ in $\mathrm{N}$-methylpyrrolidon at $175^{\circ} \mathrm{C}$ under a nitrogen atmosphere [6] and purified by column chromatography and recrystallization. The DMABN obtained from Aldrich, was chromatographed over $\mathrm{Al}_{2} \mathrm{O}_{3}$ (neutral, Machery and Nagel) with toluene as the eluent. An analysis of the impurity separated in this manner revealed its identity as DMBA, the synthetic precursor of the commercial DMABN. The purified material was then zonerefined by using an Enraf-Nonius Rapid Zonerefiner. The crystal fluorescence spectra of this purified material were essentially the same as those obtained previously [1].

The crystals of the synthetic DMABN were grown from an $n$-hexane/diethyl ether mixture $(10: 1)$. The fluorescence spectra of the crys- tals, contained in a quartz tube purged with nitrogen, were measured with a quantum-corrected Shimadzu RF-5000PC spectrofluorometer. The fluorescence decay times were determined with a picosecond laser single-photon counting (SPC) setup consisting of an argon ion laser (Coherent Sabre Innova), a dye laser (Coherent 702-1CD; Rhodamine 6G) and a frequency doubler $\left(\mathrm{LiIO}_{3}\right.$, $297 \mathrm{~nm}$ ). This setup and the analysis procedure of the fluorescence decays have been described elsewhere [7]. The instrument response function has a half-width of $25 \mathrm{ps}$ and the time resolution is estimated at around 3 ps.

\section{Results and discussion}

\subsection{Fluorescence spectra of $D M A B N$ single crys- tals}

The fluorescence spectrum of a DMABN crystal at $24{ }^{\circ} \mathrm{C}$ (Fig. 1a) consists of a single emission band with a maximum $\tilde{v}^{\max }$ at $27500 \mathrm{~cm}^{-1}$ and a shape similar to that measured in solution [8]. The crystal fluorescence spectrum of DMABN undergoes a blue-shift upon cooling, to $\tilde{v}^{\max }=28230$ $\mathrm{cm}^{-1}$ at $-112{ }^{\circ} \mathrm{C}$ (Fig. 1b). Between -60 and $-70{ }^{\circ} \mathrm{C}, \tilde{v}^{\max }$ increased sharply from 27810 to $28090 \mathrm{~cm}^{-1}$, whereas such an irregularity is not seen around $-40{ }^{\circ} \mathrm{C}$ where a solid-solid phase transition has been reported $[1,2]$. Below $-70{ }^{\circ} \mathrm{C}$, the single crystal was transformed into powder. With a microcrystalline sample of DMABN, an increase in $\tilde{v}^{\max }$ took place from $27800 \mathrm{~cm}^{-1}$ at -41 ${ }^{\circ} \mathrm{C}$ to $28010 \mathrm{~cm}^{-1}$ at $-43{ }^{\circ} \mathrm{C}$. These results show that the onset of the solid-solid phase transition may occur over a temperature range from -40 to $-70{ }^{\circ} \mathrm{C}$, depending on the nature of the particular crystal sample.

From the absence of dual emission it is concluded that the crystal fluorescence of DMABN originates from an LE state. An additional redshifted band is not observed, in contrast to what has been reported in the literature [1,2]. The observation of an LE emission band and a singleexponential fluorescence decay is in line with the presence of one molecular conformer in DMABN crystals $[2,9]$. 


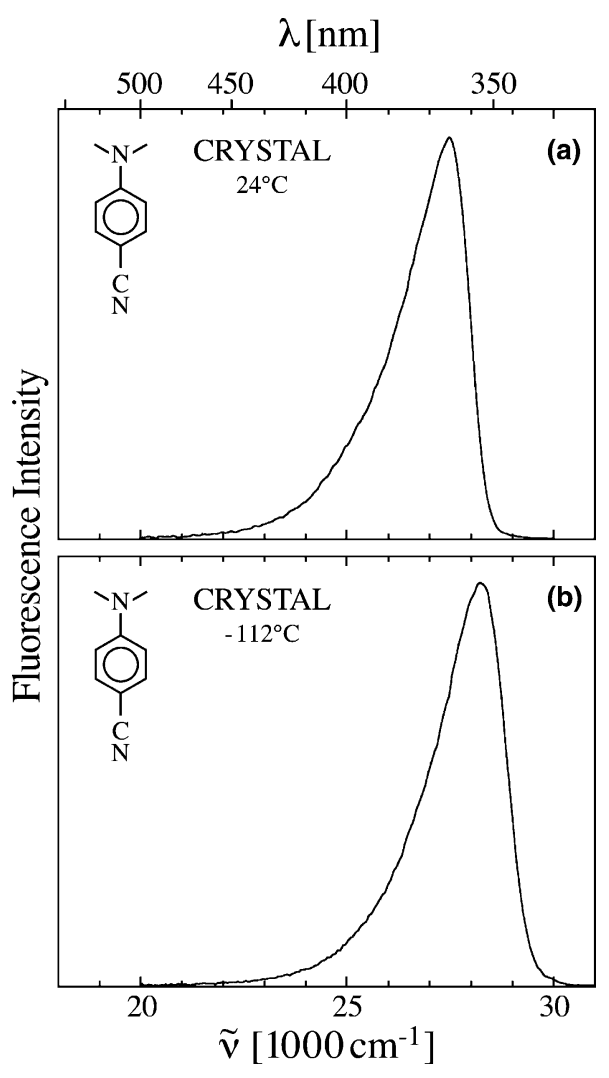

Fig. 1. Fluorescence spectra of crystalline 4-(dimethylamino)benzonitrile (DMABN) at: (a) $24^{\circ} \mathrm{C}$ and (b) $-112{ }^{\circ} \mathrm{C}$. The emission consists of a single band originating from a locally excited (LE) state. Excitation wavelength: $310 \mathrm{~nm}$.

\subsection{DMABN crystal fluorescence decays as a function of temperature}

The fluorescence decay of a single crystal of DMABN at $25{ }^{\circ} \mathrm{C}$ can under optimal conditions (see below) be represented by a single exponential, with a decay time $\tau_{1}$ of 4.25 ns, see Fig. 3a. Decay times of the same order of magnitude, between 4.0 and $4.6 \mathrm{~ns}$, are observed over the temperature range between room temperature and $-110{ }^{\circ} \mathrm{C}$ in the case of single crystals (Fig. 2b) as well as microcrystals of DMABN.

With some crystals and after prolonged laser irradiation, an additional minor (a few percent) second decay component of around $0.5 \mathrm{~ns}$ is required to achieve an adequate fitting of the decay curve. This additional time does not show a reg-
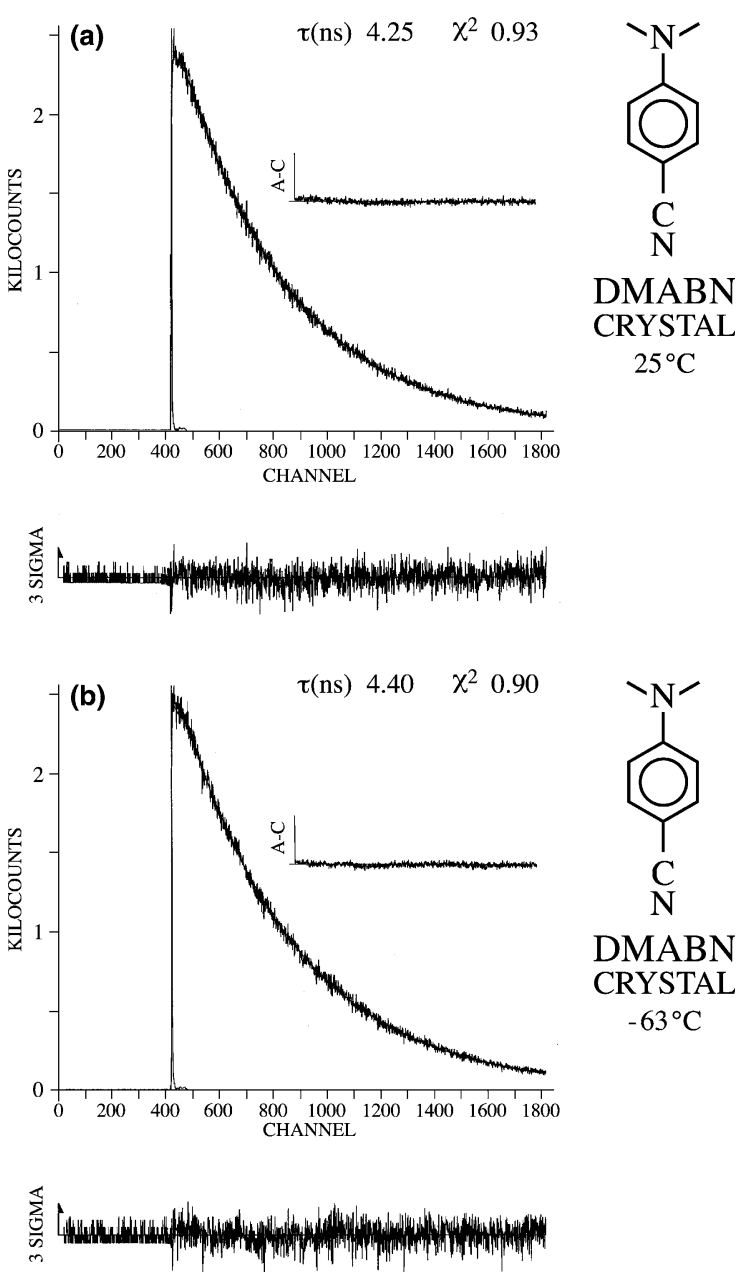

Fig. 2. Single-exponential LE fluorescence response functions with decay time $\tau$ of crystalline 4-(dimethylamino)benzonitrile (DMABN) at: (a) $26{ }^{\circ} \mathrm{C}$ and (b) $-63{ }^{\circ} \mathrm{C}$. The weighted deviations, expressed in $\sigma$ (expected deviations), the autocorrelation functions A-C, and the values for $\chi^{2}$ are also indicated. Excitation wavelength: $297 \mathrm{~nm}$. Emission wavelength: (a) $370 \mathrm{~nm}$ and (b) $365 \mathrm{~nm}$.

ular temperature dependence. In the measurements with crystalline DMABN (single crystals as well as microcrystals), the decay time $\tau$ showed a sharp decrease from 4.4 to $4.1 \mathrm{~ns}$ over a temperature interval between -60 and $-70{ }^{\circ} \mathrm{C}$, an indication for the occurrence of a solid-solid phase transition. A similar observation was made for the fluorescence emission maxima, as described in the previous section. It should again be noted that 
earlier such a phase transition was found at around $-40{ }^{\circ} \mathrm{C}[2,9]$.

The observation with crystals of synthetic DMABN of one LE emission band and singleexponential fluorescence decay over a large temperature range, shows that ICT does not occur under these conditions. The singlet excited state structure of powdered DMABN determined by picosecond X-ray diffraction [10] therefore refers to the LE state and does not provide information on the structure of the ICT state, neither planar [11] nor twisted [12].

\subsection{Fluorescence of DMABN crystals doped with $D M B A$}

Addition of a small amount $(0.8 \%)$ of 4 -(dimethylamino)benzaldehyde (DMBA) to the synthetic DMABN has two effects. First, an additional red-shifted structured emission band appears in the fluorescence spectrum of the crystal (Fig. 3) and, secondly, the overall intensity of the fluorescence decreases by a factor of around 50 relative to that of crystals of pure DMABN (Figs. 1 and 2).

The total fluorescence spectrum in Fig. 3 is similar to that of crystals grown from commercial DMABN $[1,2]$. The presence of the structured red-

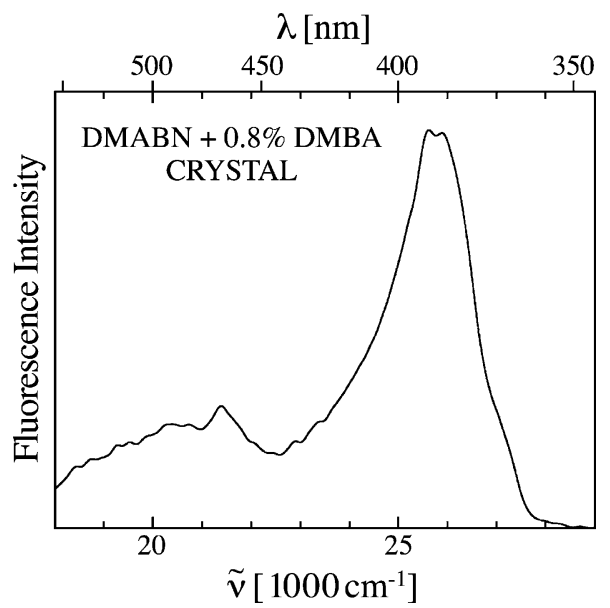

Fig. 3. Fluorescence spectrum at $25{ }^{\circ} \mathrm{C}$ of crystalline 4-(dimethylamino)benzonitrile (DMABN) doped with $0.8 \%$ of 4-(dimethylamino)benzaldehyde (DMBA). The structured part of the spectrum below $22000 \mathrm{~cm}^{-1}$ is attributed to DMBA, see text. Excitation wavelength: $330 \mathrm{~nm}$.
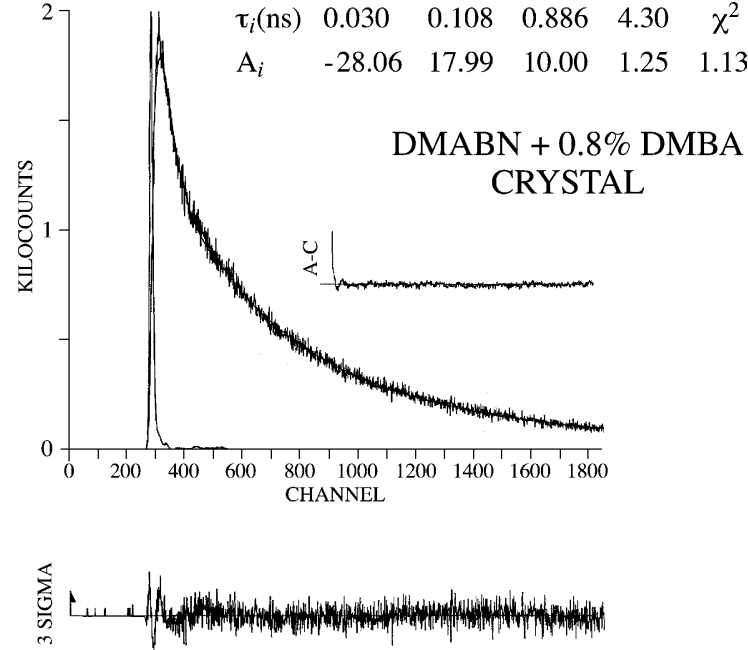

Fig. 4. Multiexponential fluorescence response function at $25{ }^{\circ} \mathrm{C}$ of crystalline 4-(dimethylamino)benzonitrile (DMABN) doped with 0.8 percent of 4-(dimethylamino)benzaldehyde (DMBA). The decay times $\tau_{\mathrm{i}}$ and their pre-exponential factors $A_{\mathrm{i}}$ are given (Eq. (1)). The shortest decay time $\left(\tau_{4}\right)$ is listed first. Excitation wavelength: $297 \mathrm{~nm}$. Emission wavelength: $385 \mathrm{~nm}$. See the caption of Fig. 2.

shifted emission band in these spectra as well as the relatively small crystal fluorescence intensity (see above) is therefore attributed to the presence of a DMBA impurity, which apparently could not be removed by the purification procedures employed.

The picosecond fluorescence decays of the DMABN crystals doped with DMBA at $25{ }^{\circ} \mathrm{C}$ (Fig. 4) can be fitted with four exponentials (Eq. (1)), with the decay times of $0.030 \mathrm{~ns}(5 \%)$, $0.108 \mathrm{~ns}(11 \%), 0.886 \mathrm{~ns}(52 \%)$ and $4.30 \mathrm{~ns}(32 \%)$, based on the absolute values of the amplitude ratios, see Fig. 4. The multiexponential character of the doped DMABN crystals is similar to that observed with the commercial crystalline DMABN [1]

$$
\begin{aligned}
i_{\mathrm{f}}= & A_{4} \exp \left(-t / \tau_{4}\right)+A_{3} \exp \left(-t / \tau_{3}\right) \\
& +A_{2} \exp \left(-t / \tau_{2}\right)+A_{1} \exp \left(-t / \tau_{1}\right) .
\end{aligned}
$$

\section{Conclusions}

Crystals of DMABN synthesized from 4-bromo- $N, N$-dimethylaniline show a fluorescence spectrum consisting of a single LE emission band, 
without any evidence for ICT fluorescence. This is the case over an extended temperature range from 25 down to $-112^{\circ} \mathrm{C}$. The crystal fluorescence decays are single exponential. Addition of a small amount $(0.8 \%)$ of DMBA to the synthetic DMABN leads to a substantial quenching by a factor of around 50 of the crystal fluorescence and the appearance of an additional red-shifted structured emission. The overall fluorescence spectrum is similar to that of DMABN crystals previously reported in the literature, which is in support of our interpretation that impurity quenching by DMBA occurred in these spectra. The present results confirm that ICT does not take place with crystalline DMABN and that therefore conclusions on the molecular structure of its ICT state cannot be based on picosecond X-ray studies of these crystals.

\section{Acknowledgements}

A.D. acknowledges support from the Hungarian Science Foundation (OTKA Project No. T
33102). Wilfried Bosch is thanked for the synthesis of DMABN.

\section{References}

[1] S. Druzhinin, A. Demeter, K.A. Zachariasse, Chem. Phys. Lett. 347 (2001) 421.

[2] G.B. Jameson, B.M. Sheikh-Ali, R.G. Weiss, Acta Cryst. B 50 (1994) 703.

[3] K.W. Benz, H.C. Wolf, Z. Naturforsch. 19 (1964) 177.

[4] M. Pope, C. Swenberg, Electronic Processes in Organic Crystals and Polymers, second ed., Oxford University Press, Oxford, 1999.

[5] Unpublished results.

[6] R. Reynaud, Bull. Soc. Chim. Fr. (1967) 2686.

[7] Yu.V. Il'ichev, W. Kühnle, K.A. Zachariasse, J. Phys. Chem. A 102 (1998) 5670.

[8] S.I. Druzhinin, Y.-B. Jiang, A. Demeter, K.A. Zachariasse, Phys. Chem. Chem. Phys. 3 (2001) 5213.

[9] A. Heine, R. Herbst-Irmer, D. Stalke, W. Kühnle, K.A. Zachariasse, Acta Cryst. B 50 (1994) 363.

[10] S. Techert, F. Schotte, M. Wulff, Phys. Rev. Lett. 86 (2001) 2030.

[11] K.A. Zachariasse, Chem. Phys. Lett. 320 (2000) 8.

[12] C. Day, Phys. Today 19 (1994) 363 (54 (March 2001)). 